\title{
Publisher's Note: Hořava-Lifshitz bouncing Bianchi IX universes: A dynamical system analysis [Phys. Rev. D 96, 103532 (2017)]
}

Rodrigo Maier and Ivano Damião Soares

(Q) (Received 6 February 2018; published 20 February 2018)

DOI: $10.1103 /$ PhysRevD.97.049902

This paper was published online on 21 November 2017 with typographical errors in Eqs. (11), (12), (15), (16), and (23), a typographical error in the unnumbered equation on page 4, and a typographical error in text in the fourth line, left-hand column, on page 4.

Equations (11) should read as

$$
K_{i j}=\frac{1}{2 N} \dot{h}_{i j}=\frac{1}{N}(-M \dot{M},-Q \dot{Q},-R \dot{R})
$$

and

$$
K^{i j}=h^{i m} h^{j n} K_{m n}=\frac{1}{N}\left(-\frac{\dot{M}}{M^{3}},-\frac{\dot{Q}}{Q^{3}},-\frac{\dot{R}}{R^{3}}\right) .
$$

Equation (12) should read as

$$
{ }^{(3)} R=-\frac{1}{2 M^{2} Q^{2} R^{2}}\left[M^{4}+Q^{4}+R^{4}-\left(R^{2}-Q^{2}\right)^{2}-\left(R^{2}-M^{2}\right)^{2}-\left(M^{2}-Q^{2}\right)^{2}\right] .
$$

On page 4, the non-numbered equation above Eq. (15) should read as

$$
\mathcal{L} \propto \mathcal{K}-V .
$$

Also on page 4 , the fourth line of the left-hand column should read "where the kinetic part $\mathcal{K}$ is given by...". Equation (15) should read as

$$
\mathcal{K}=\frac{M Q R}{N}\left[(1-\lambda)\left(\frac{\dot{M}^{2}}{M^{2}}+\frac{\dot{Q}^{2}}{Q^{2}}+\frac{\dot{R}^{2}}{R^{2}}\right)-2 \lambda\left(\frac{\dot{M} \dot{Q}}{M Q}+\frac{\dot{Q} \dot{R}}{Q R}+\frac{\dot{M} \dot{R}}{M R}\right)\right] .
$$

Equation (16) should read as

$$
V=N(M Q R)\left[{ }^{(3)} R+2 \Lambda+U_{\mathrm{HL}}\right]+2 N\left(E_{0}+\frac{E_{r}}{(M Q R)^{1 / 3}}\right) .
$$

Equation (23) should read as

$$
V(M)=\frac{3}{2} M-2 \Lambda M^{3}-\frac{3}{4} \frac{A_{2}}{M}+\frac{3}{8} \frac{A_{3}}{M^{3}} .
$$

The equations and text have been corrected as of 14 February 2018. The equations and text are incorrect in the printed version of the journal. 